\title{
Variance estimation in nonparametric regression with jump discontinuities
}

\author{
Wenlin Dai and Tiejun Tong* \\ Department of Mathematics, Hong Kong Baptist University, Kowloon Tong, Hong Kong
}

(Received 21 September 2012; accepted 6 September 2013)

\begin{abstract}
Variance estimation is an important topic in nonparametric regression. In this paper, we propose a pairwise regression method for estimating the residual variance. Specifically, we regress the squared difference between observations on the squared distance between design points, and then estimate the residual variance as the intercept. Unlike most existing difference-based estimators that require a smooth regression function, our method applies to regression models with jump discontinuities. Our method also applies to the situations where the design points are unequally spaced. Finally, we conduct extensive simulation studies to evaluate the finite-sample performance of the proposed method and compare it with some existing competitors.
\end{abstract}

Keywords: difference-based estimator; jump point; nonparametric regression; non-uniform design; pairwise regression; residual variance

\section{Introduction}

Consider a nonparametric regression model with jump discontinuities

$$
y_{i}=g\left(x_{i}\right)+h\left(x_{i}\right)+\varepsilon_{i}, \quad 1 \leq i \leq n,
$$

where $y_{i}$ are observations, $g$ is a continuous function, $h$ is a step function, and $\varepsilon_{i}$ are independent and identically distributed random errors with zero mean and variance $\sigma^{2}$. To be specific, we write the step function $h$ as

$$
h(x)=\sum_{j=1}^{p} c_{j} I\left(x>t_{j}\right),
$$

where $p$ is the number of jumps, $I(\cdot)$ is the identify function with value 1 when $x>t_{j}$ and value 0 otherwise, and $c_{j}$ are the magnitudes of jumps at the jump points $t_{j} \in(0,1)$, respectively. Note that $g+h$ is the mean function.

Model (1) has wide applications in statistical process control [18], piecewise linear regression $[1,9,10]$, image processing $[8,12,17]$, and other related areas. It has also been applied to many

\footnotetext{
*Corresponding author. Email: tongt@hkbu.edu.hk
} 
real data examples such as the Nile river discharge data [2], the stock market return data [25], the sea-level pressure data [19], and the infants growth data [14]. There is an abundant literature for analysing model (1) including the detection and estimation of the number, positions, and magnitudes of jump points [4,11,13,26,27].

This paper considers the estimation of the residual variance $\sigma^{2}$ in model (1). Needless to say, an accurate estimate of $\sigma^{2}$ is very important in regression models with jump discontinuities. Usually, one applies a two-step procedure to estimate $\sigma^{2}$ in such models. The first step is to estimate the positions of change points and then divide the mean function into several continuous sections accordingly. The second step is to estimate the residual variance within each individual section and then use them to make a final estimate of $\sigma^{2}$. Note that one may apply the residual-based methods [7] or apply the difference-based methods $[13,26]$ to estimate the residual variance within each individual section.

Apart from the above, Müller and Stadtmüller [14] proposed a single-step method for estimating $\sigma^{2}$ in model (1). Consider the equally spaced design where $x_{i}=i / n, i=1, \ldots, n$. Let

$$
z_{k}=\sum_{i=1}^{n-L} \frac{\left(y_{i+k}-y_{i}\right)^{2}}{[2(n-L)]}
$$

where $k=1, \ldots, L$ with $L=L(n) \geq 1$. Under certain conditions on the mean function and the bandwidth $L$, Müller and Stadtmüller showed that

$$
E\left(z_{k}\right) \approx \sigma^{2}+\gamma l_{k}+\delta l_{k}^{2}
$$

where $l_{k}=k /(n-L), \gamma=\sum_{j=1}^{p-1}\left(c_{j+1}-c_{j}\right)^{2} / 2$ is the amount of discontinuity in the data, and $\delta=\int_{0}^{1}\left[g^{\prime}(x)\right]^{2} \mathrm{~d} x / 2+\sum_{j=1}^{p-1} g^{\prime}\left(t_{j+1}\right)\left(c_{j+1}-c_{j}\right)$ is the measurement of the interaction between continuous and discontinuous parts. By Equation (2), they fitted a quadratic regression that regresses $z_{k}$ on $l_{k}$ and then estimate the residual variance as the intercept. Specifically, they estimated $\sigma^{2}$ by

$$
\hat{\sigma}_{\mathrm{MS}}^{2}=\frac{3 \sum_{k=1}^{L}\left(3 L^{2}+3 L+2-6(2 L+1) k+10 k^{2}\right) z_{k}}{2 L(L-1)(L-2)} .
$$

This method does not require an estimate of the positions of change points and is popular in practice.

Note that $z_{k}$ only uses the first $n-L$ pairs of observations for performing the quadratic regression. Ignoring the last $L-k$ terms can make $z_{k}$ a less efficient representation for $\sigma^{2}$, especially when $L-k$ is large. In addition, Müller and Stadtmüller [14] required that $\min _{1 \leq i \leq p-1}\left(t_{i+1}-t_{i}\right) \geq$ $2 L / N$ for the possibility of change-points separation. In the special case when $\gamma=0$, i.e. when $h(x)=0$, Tong et al. [23] have demonstrated that the least-squares estimator in Tong and Wang [24] provides a smaller mean squared error (MSE) than $\hat{\sigma}_{\mathrm{MS}}^{2}$. In addition, the equally spaced design condition in Müller and Stadtmüller [14] is somewhat strong and has limited the practical use of $\hat{\sigma}_{\text {MS }}^{2}$.

In this paper, we propose a pairwise regression method for estimating $\sigma^{2}$ in model (1). Specifically, we regress the squared difference between observations on the squared distance between design points, and then estimate the residual variance as the intercept. Our method generalizes the existing methods from the following perspectives: (1) it does not require to estimate the positions of change points compared with the two-step estimators in the literature; (2) it does not require to estimate the discontinuity parameter $\gamma$ compared with the single-step estimator in Müller and Stadtmüller [14]; and (3) it also applies to the settings where the design points are unequally spaced. 
The remainder of the paper is organized as follows. In Section 2.1, we review the differencebased methods in estimating the residual variance in continuous nonparametric regression. In Section 2.2, we propose a pairwise regression method that extends the least-squares estimator in Tong and Wang [24] to unequally spaced designs. In Section 2.3, we further extend the proposed pairwise regression method to adaptively estimate the residual variance in nonparametric regression with jump discontinuities. In Section 3, we conduct extensive simulation studies to evaluate the finite-sample performance of the proposed method with some existing competitors. We then apply the proposed method to a real data example in Section 4 and conclude the paper in Section 5 with some discussions.

\section{Main results}

\subsection{Difference-based estimators}

In the special case when $h(x)=0$, model (1) reduces to

$$
y_{i}=g\left(x_{i}\right)+\varepsilon_{i}, \quad 1 \leq i \leq n .
$$

Under model (4), there are many difference-based methods in the literature for estimating $\sigma^{2}$. Assume that $0 \leq x_{1} \leq \cdots \leq x_{n} \leq 1$. von Neumann [16] and Rice [20] proposed a first-order difference-based estimator

$$
\hat{\sigma}_{\mathrm{R}}^{2}=\frac{1}{2(n-1)} \sum_{i=2}^{n}\left(y_{i}-y_{i-1}\right)^{2} .
$$

Gasser et al. [5] and Hall et al. [6] extended the idea and proposed some higher order differencebased estimators. In addition, Müller et al. [15], Tong et al. [22], and Du and Schick [3] proposed covariate-matched U-statistic estimators for the residual variance.

Apart from them, Tong and Wang [24] and Tong et al. [23] proposed a variation of the difference-based estimator in nonparametric regression. Let $x_{i}=i / n$ and $s_{k}=\sum_{i=k+1}^{n}\left(y_{i}-y_{i-k}\right)^{2} /[2(n-k)]$. Suppose that $g$ has a bounded first derivative. Tong and Wang [24] showed that for any fixed $m=o(n)$

$$
E\left(s_{k}\right) \approx \sigma^{2}+d_{k} J, \quad k=1, \ldots, m,
$$

where $d_{k}=k^{2} / n^{2}$ and $J=\int_{0}^{1}\left[g^{\prime}(x)\right]^{2} \mathrm{~d} x / 2$. By Equation (5), they regressed $s_{k}$ on $d_{k}$ and then estimated the residual variance as the intercept. Specifically, their least-squares estimator is given as

$$
\hat{\sigma}_{\mathrm{TW}}^{2}=\sum_{k=1}^{m} w_{k} s_{k}-\hat{\beta} \bar{d}_{w},
$$

where $N_{1}=m n-m(m+1) / 2, \quad w_{k}=(n-k) / N_{1}, \quad \bar{d}_{w}=\sum_{k=1}^{m} w_{k} s_{k}, \quad$ and $\hat{\beta}=\sum_{k=1}^{m} w_{k} s_{k}$ $\left(d_{k}-\bar{d}_{w}\right) / \sum_{k=1}^{m} w_{k}\left(d_{k}-\bar{d}_{w}\right)^{2}$.

Recall that $\hat{\sigma}_{\text {TW }}^{2}$ is developed under model (4) with a continuous mean function. When $h(x) \neq 0$, $\hat{\sigma}_{\text {TW }}^{2}$ may not perform well in model (1). To illustrate this, we consider the following regression model with a single jump at $t=0.5$

$$
y_{i}=g\left(x_{i}\right)+c I\left(x_{i}>0.5\right)+\varepsilon_{i}, \quad c>0 .
$$




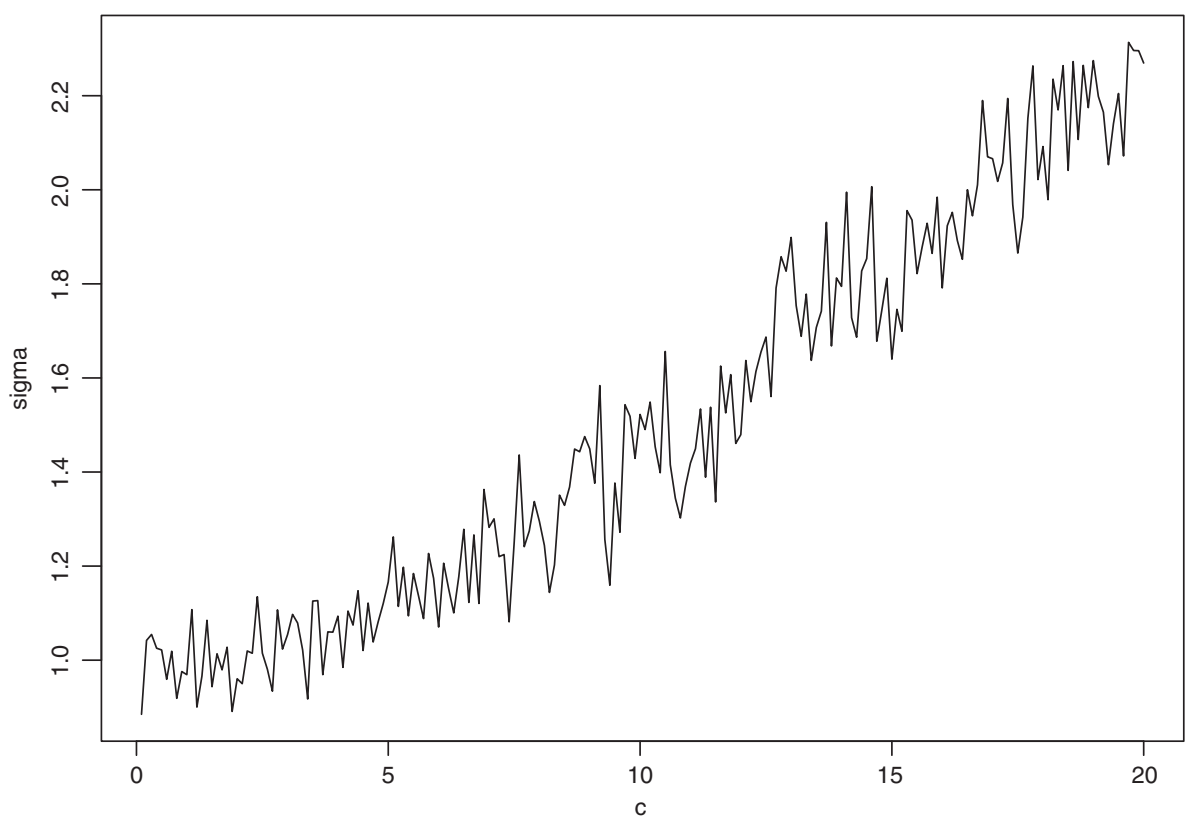

Figure 1. The estimated $\hat{\sigma}^{2}$ corresponding to different $c$ values.

Assume that $J$ and $c$ are both finite values. We have

$$
\begin{aligned}
E\left(s_{k}\right)= & \sigma^{2}+\frac{1}{2(n-k)} \sum_{i=k+1}^{n}\left\{\left[g\left(x_{i}\right)+c I\left(x_{i}>0.5\right)\right]\right. \\
& \left.-\left[g\left(x_{i-k}\right)+c I\left(x_{i-k}>0.5\right)\right]\right\}^{2} \\
= & \sigma^{2}+\left[d_{k} J+o\left(\frac{k^{2}}{n^{2}}\right)\right]+\left[\frac{k}{n} c^{2}+o\left(\frac{k}{n}\right)\right] .
\end{aligned}
$$

Note that the bias owing to the jump, $(k / n) c^{2}$, dominates the bias owing to the continuous function, $d_{k} J=(k / n)^{2} J$. This implies that $\hat{\sigma}_{\mathrm{TW}}^{2}$ may suffer a severe bias for estimating $\sigma^{2}$, especially when $c$ is large.

For a visualization of the bias pattern along with the $c$ value, consider $g(x)=5 x(1-x)$ and $h(x)=c I(x>0.5)$ with $0<c<20$. We let $n=100, m=10$, and $\sigma^{2}=1$ throughout the simulations. The estimated variance against the $c$ value is plotted in Figure 1. We observe that $\hat{\sigma}_{\mathrm{TW}}^{2}$ increases rapidly as $c$ increases. As a consequence, $\hat{\sigma}_{\mathrm{TW}}^{2}$ does not provide a satisfactory performance in this example.

\subsection{Pairwise regression}

Recall that the least-squares estimator in Tong and Wang [24] only applies to the equally spaced design. This has largely restricted the usage of their method in practice. In this section, we introduce a pairwise regression method for estimating the residual variance that extends the least-squares estimator from the equally spaced design to unequally spaced designs.

Let $s_{i j}=\left(y_{j}-y_{i}\right)^{2} / 2$ be the half squared differences and $d_{i j}=\left(x_{j}-x_{i}\right)^{2}$ be the corresponding squared distances for any $1 \leq i<j \leq n$. Let $d=o(1)$ be the bandwidth. We collect all $d_{i j}$ values 
that satisfy $d_{i j} \leq d$. For ease of notation, let $A=\left\{(i, j): d_{i j} \leq d, 1 \leq i<j \leq n\right\}$ and $N=\#(A)$ be the total number of pairs in $A$. Correspondingly, we collect the $s_{i j}$ values for all $(i, j) \in A$.

Note that $E\left(s_{i j}\right)=\sigma^{2}+\left(g\left(x_{j}\right)-g\left(x_{i}\right)\right)^{2} / 2$. When $g$ is a linear function with slope $\psi$, we have $E\left(s_{i j}\right)=\sigma^{2}+d_{i j} \psi^{2} / 2$. In view of this, for the paired data $\left\{\left(d_{i j}, s_{i j}\right):(i, j) \in A\right\}$ with $d=o(1)$, we fit a simple linear regression model that regresses $s_{i j}$ directly on $d_{i j}$

$$
s_{i j}=\alpha+d_{i j} \beta+\eta_{i j}
$$

We then use the ordinary least-squares method to estimate $\sigma^{2}$ using the fitted intercept. This leads to

$$
\hat{\sigma}^{2}=\hat{\alpha}=\frac{\sum_{A}\left(S_{2}-S_{1} d_{i j}\right) s_{i j}}{N S_{2}-S_{1}^{2}},
$$

where $S_{1}=\sum_{A} d_{i j}$ and $S_{2}=\sum_{A} d_{i j}^{2}$. We refer to Equation (9) as a pairwise regression estimator.

Let $c_{i j}=S_{2}-S_{1} d_{i j}$ and $y=\left(y_{1}, \ldots, y_{n}\right)^{\mathrm{T}}$. The estimator (9) has a quadratic form $\hat{\sigma}^{2}=$ $y^{\mathrm{T}} M y / \operatorname{tr}(M)$, where $M$ is an $n \times n$ symmetric matrix with upper triangular elements

$$
m_{i j}= \begin{cases}\sum_{(i, j) \in A_{k}} \frac{c_{i j}}{2}, & 1 \leq i=j=k \leq n, \\ -\frac{c_{i j}}{2}, & (i, j) \in A, \\ 0, & \text { otherwise, }\end{cases}
$$

where $A_{k}=\{(i, j): i=k$ or $j=k,(i, j) \in A\}$ with $k=1,2, \ldots, n$.

In what follows we draw some connection between the pairwise regression estimator $\hat{\sigma}^{2}$ and the least-squares estimator $\hat{\sigma}_{\mathrm{TW}}^{2}$. Let $x_{i}=i / n$ and $d=m^{2} / n^{2}$. Then $N=N_{1}=m n-m(m+1) / 2$ and $d_{i j}=d_{j-i}$. Also, it is easy to verify that $S_{1}=N \bar{d}_{w}, S_{2}=N \sum_{k=1}^{m} w_{k} d_{k}^{2}, \sum_{A} s_{i j}=N \sum_{k=1}^{m} w_{k} s_{k}$, and $\sum_{A} d_{i j} s_{i j}=N \sum_{k=1}^{m} w_{k} d_{k} s_{k}$. With the above equalities, we have

$$
\begin{aligned}
\hat{\sigma}^{2} & =\frac{S_{2} \sum_{A} s_{i j}-S_{1} \sum_{A} d_{i j} s_{i j}}{N S_{2}-S_{1}^{2}} \\
& =\frac{\sum_{k=1}^{m} w_{k} d_{k}^{2} \sum_{k=1}^{m} w_{k} s_{k}-\bar{d}_{w} \sum_{k=1}^{m} w_{k} d_{k} s_{k}}{\sum_{k=1}^{m} w_{k} d_{k}^{2}-\bar{d}_{w}^{2}} \\
& =\sum_{k=1}^{m} w_{k} s_{k}-\bar{d}_{w} \hat{\beta} \\
& =\hat{\sigma}_{\mathrm{TW}}^{2} .
\end{aligned}
$$

This shows that when the design points are equally spaced, $\hat{\sigma}^{2}$ and $\hat{\sigma}_{\mathrm{TW}}^{2}$ are equivalent to each other. From this point of view, we conclude that the pairwise regression estimator (9) generalized the least-squares estimator $\hat{\sigma}_{\mathrm{TW}}^{2}$ from the equally spaced design to a general design.

\subsection{Adaptive pairwise regression}

As mentioned, most existing difference-based estimators were developed under model (4). In this section, we show that the pairwise regression method in Section 2.2 can be readily extended to model (1) with jump discontinuities.

To apply the pairwise regression to models with jump discontinuities, we revisit the simple regression model presented in Equation (7). Let $O=\left\{(i, j): 0.5 \in\left(x_{i}, x_{j}\right]\right\}$ be the pairs of design 
points that cross the jump point. By Equation (9), we have

$$
\begin{aligned}
E\left(\hat{\sigma}^{2}\right) & =\frac{\sum_{A \backslash O}\left(S_{2}-S_{1} d_{i j}\right) E\left(s_{i j}\right)}{N S_{2}-S_{1}^{2}}+\frac{\sum_{O}\left(S_{2}-S_{1} d_{i j}\right) E\left(s_{i j}\right)}{N S_{2}-S_{1}^{2}} \\
& =\frac{\sum_{A \backslash O}\left(S_{2}-S_{1} d_{i j}\right)\left(\sigma^{2}+O\left(m^{2} / n^{2}\right)\right)}{N S_{2}-S_{1}^{2}}+\frac{\sum_{O}\left(S_{2}-S_{1} d_{i j}\right)\left(\sigma^{2}+c^{2} / 2+O(m / n)\right)}{N S_{2}-S_{1}^{2}} \\
& =\frac{\sum_{A}\left(S_{2}-S_{1} d_{i j}\right)}{N S_{2}-S_{1}^{2}} \sigma^{2}+\frac{\sum_{O}\left(S_{2}-S_{1} d_{i j}\right)}{N S_{2}-S_{1}^{2}}\left(\frac{c^{2}}{2}+O\left(\frac{m}{n}\right)\right) \\
& =\sigma^{2}+\frac{\sum_{O}\left(S_{2}-S_{1} d_{i j}\right)}{N S_{2}-S_{1}^{2}} \frac{c^{2}}{2}+O\left(\frac{m^{2}}{n^{2}}\right)
\end{aligned}
$$

where

$$
\frac{\sum_{A}\left(S_{2}-S_{1} d_{i j}\right)}{N S_{2}-S_{1}^{2}}=1 \quad \text { and } \quad \frac{\sum_{O}\left(S_{2}-S_{1} d_{i j}\right)}{N S_{2}-S_{1}^{2}}=O\left(\frac{m}{n}\right) .
$$

By Equation (10), to obtain a good estimate of $\sigma^{2}$, it is clear that the pairs in $O$ should be excluded from the regression to eliminate the bias. Otherwise, given that the quantity $c$ is large, the extra bias introduced by the jump can be very severe.

In what follows, we examine how excluding the pairs in $O$ takes effect on the MSE of the estimator. We will also suggest ways to exclude certain pairs of data from the pairwise regression. Let $z_{i j}=y_{j}-y_{i}$ for any $1 \leq i<j \leq n$. For $d=o(1)$, we have $E\left(z_{i j}\right) \rightarrow 0$ for $(i, j) \in O$ and $E\left(z_{i j}\right) \rightarrow c$ for $(i, j) \in A \backslash O$. Whereas for any $(i, j) \in A$, $\operatorname{var}\left(z_{i j}\right)=2 \sigma^{2}$. To visualize the discrepancy between the two groups of $z_{i j}$, we consider $c=0,2$ and 5 for the example in Section 2.1. All other settings are kept the same as before except that now $\sigma=0.5$.

We plot the histograms of the simulated $z_{i j}$ values in the first column of Figure 2. When the mean function is continuous (i.e. $c=0$ ), the histogram is unimodal and almost symmetric around zero. When $c$ increases, the histogram tends to be right-skewed and eventually separates to two disjoint sections, one consisting of the pairs without jump and the other consisting of the pairs with jump. To eliminate the impact of the jump on the variance estimation, we can treat the extremely large $\left|z_{i j}\right|$ values, or correspondingly the extremely large $s_{i j}$ values, as outliers and exclude them in the pairwise regression.

Ideally, none of the $z_{i j}$ values should be detected as outliers when the mean function is continuous. When $c$ is non-zero, to reduce the bias or essentially to gain a small MSE we may wish to drop the pairs that cross the jump point. As an illustration, we also plot in Figure 2 the simulated MSE against the number of pairs dropped for the three $c$ values, respectively. It suggests to drop few pairs for $c=0$, drop around 40 pairs for $c=2$, and drop around 55 pairs for $c=5$ for estimating the residual variance with a minimum MSE. Finally, it is interesting to point out that for an equally spaced design with $m=10$, there is a total of $m(m+1) / 2=55$ pairs across the jump point.

In what follows, we suggest two practical rules that identify certain $z_{i j}$ values as outliers and then exclude them from the pairwise regression. The resulting methods are referred to as adaptive pairwise regression estimators.

\subsubsection{Box plot method}

The first method uses the box plot to detect certain $z_{i j}$ values as outliers. Let $Q_{\mathrm{L}}\left(\left\{z_{i j}\right\}\right)$ and $Q_{\mathrm{U}}\left(\left\{z_{i j}\right\}\right)$ denote the lower quartile and the upper quartile of the observed $z_{i j}$ values within the bandwidth, respectively. Follow the form of Sim et al. [21], we define $\mathrm{LB}=Q_{\mathrm{L}}\left(\left\{z_{i j}\right\}\right)-C \cdot \mathrm{IQR}$ and $\mathrm{UB}=$ $Q_{\mathrm{U}}\left(\left\{z_{i j}\right\}\right)+C \cdot \mathrm{IQR}$, where IQR $=Q_{\mathrm{U}}\left(\left\{z_{i j}\right\}\right)-Q_{\mathrm{L}}\left(\left\{z_{i j}\right\}\right)$ is the interquartile range and $C$ is an 

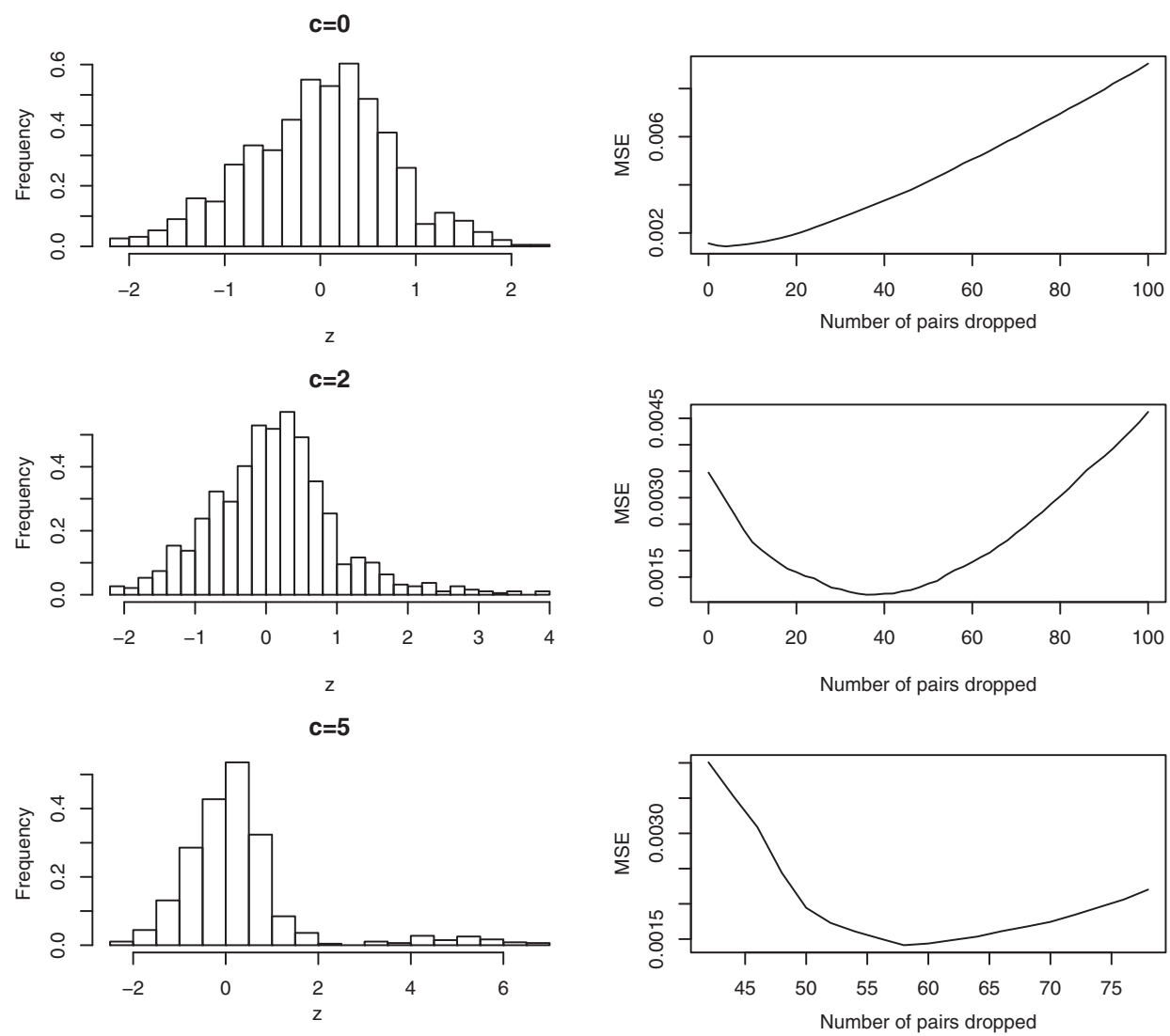

Figure 2. The histogram of $z_{i j}$ and the change of MSE against the number of pairs dropped, where $c=0,2$ and 5 , respectively.

adjustment factor. Here, we assign a value of 2 or 3 to $C$. We then identify $z_{i j}$ as an outlier if $z_{i j} \in(-\infty, \mathrm{LB})$ or $z_{i j} \in(\mathrm{UB}, \infty)$. We refer to the estimator by the box plot method as $\hat{\sigma}_{\text {box }}^{2}$.

\subsubsection{Cross-validation method}

Note that the bandwidth $d$ is also critical to the variance estimation. Our second method uses a $V$-fold cross-validation (CV) approach to simultaneously choose the bandwidth $d$ and the adjustment factor $C$. Specifically, we first split the whole data set into $V$ disjoint subsamples, $S_{1}, \ldots, S_{V}$ as in Tong and Wang [24]. Second, for given $d$ and $C$, we estimate $\sigma^{2}$ by $\hat{\sigma}_{v}^{2}(d, C)$ based on the subsample $\cup_{i \neq v} S_{i}$ and the pairs with $d_{i j} \leq d$ and $z_{i j} \in[\operatorname{LB}(C), \mathrm{UB}(C)]$. Finally, we choose the optimal tuning parameters $d$ and $C$ that minimize

$$
\mathrm{CV}(d, C)=\sum_{v=1}^{V}\left[\hat{\sigma}^{2}(d, C)-\hat{\sigma}_{v}^{2}(d, C)\right]^{2}
$$

where $\hat{\sigma}^{2}(d, C)$ is the estimate of $\sigma^{2}$ based on the whole data set with pairs $d_{i j} \leq d$ and $z_{i j} \in$ $[\mathrm{LB}(C), \mathrm{UB}(C)]$. We refer to the estimator by the $\mathrm{CV}$ method as $\hat{\sigma}_{\mathrm{CV}}^{2}$. 


\section{Simulations}

In this section, we conduct extensive simulation studies to evaluate the finite-sample performance of the proposed estimators and compare them with some existing competitors.

\subsection{Equidistant design}

The first study assumes an equally spaced design. Specifically, let $x_{i}=i / n$ with $i=1, \ldots, n$. We consider the following four estimators for comparison: $\hat{\sigma}_{\mathrm{box}}^{2}, \hat{\sigma}_{\mathrm{CV}}^{2}, \hat{\sigma}_{\mathrm{MS}}^{2}$, and $\hat{\sigma}_{\mathrm{TW}}^{2}$. We consider a total of nine mean functions with combinations $g_{i}+h_{j}$ from the following functions:

$$
\begin{aligned}
& g_{1}(x)=5 x(1-x), \\
& g_{2}(x)=5 \exp (1-x), \\
& g_{3}(x)=5 \sin (2 x),
\end{aligned}
$$

and

$$
\begin{aligned}
& h_{1}(x)=4 I\left(x>\frac{\sqrt{2}}{2}\right), \\
& h_{2}(x)=3 I\left(x>\frac{\sqrt{2}}{4}\right)+4 I\left(x>\frac{\sqrt{2}}{2}\right), \\
& h_{3}(x)=0 .
\end{aligned}
$$

For each mean function, we consider $n=30,100$, and 500, ranging from small to large sample sizes, respectively, and $\sigma=0.2,0.5,1,2$, and 5 , ranging from small to large variances, respectively. Finally, for given $n$ and $\sigma$, we simulate the random errors $\varepsilon_{i}$ independently from $N\left(0, \sigma^{2}\right)$.

For each simulation setting, we generate observations and compute the estimators $\hat{\sigma}_{\mathrm{TW}}^{2}(m)$, $\hat{\sigma}_{\mathrm{MS}}^{2}(L), \hat{\sigma}_{\mathrm{box}}^{2}(d, C)$, and $\hat{\sigma}_{\mathrm{cv}}^{2}$. Note that the bandwidth $L$ in Müller and Stadtmüller [14] is not very sensitive to the estimation of $\sigma^{2}$. We consider both $L_{s}=m_{s}=n^{1 / 2}$ and $L_{t}=m_{t}=n^{1 / 3}$ as in Tong and Wang [24]. This leads to the corresponding $d$ values as $d_{s}=\left(m_{s} / n\right)^{2}$ and $d_{t}=\left(m_{t} / n\right)^{2}$. Then together with $C=2$ and 3, we have four different estimates for $\hat{\sigma}_{\text {box }}^{2}$. Recall that the CV estimator, $\hat{\sigma}_{\mathrm{cv}}^{2}$, aims to figure out the best combination between $d$ and $C$. We consider leave-one-out $\mathrm{CV}$ for $n=30$, and 10 -fold CV for $n=100$ and $n=500$, throughout the simulations.

We repeat the process 1000 times and compute the following relative MSEs, MSE/MSE ${ }_{\text {opt }}$, for each method. Here, $\mathrm{MSE}_{\mathrm{opt}}=n^{-1}\left(\gamma_{4}-1\right) \sigma^{4}$ is specified as the optimal efficiency bound of all root- $n$ consistent estimators of $\sigma^{2}$, and $\gamma_{4}=E\left(\varepsilon^{4}\right) / \sigma^{4}$. For normal errors, we have $\gamma_{4}=3$ and $\mathrm{MSE}_{\mathrm{opt}}=2 \sigma^{4} / n$. We observe that negative estimates indicated by Tong and Wang [24] and Müller and Stadtmüller [14] do appear in certain simulations, though very rarely. We replace the negative estimates with zero when calculating the relative MSEs.

\subsection{Non-equidistant design}

This section carries out simulation studies for unequally spaced designs. We generate design points from the beta distribution $\operatorname{Beta}(3,3)$. This is a bell shaped distribution on $[0,1]$ with a mode at 0.5 . Also for simplicity, we consider only three mean functions $g_{3}+h_{i}$, where the first two functions are discontinuous and the last one is continuous. All other settings are kept the same as those in Section 3.1. 
Finally, recall that Müller and Stadtmüller [14] and Tong and Wang [24] do not apply to unequally spaced designs. We thus omit both the estimators but add in the pairwise regression estimator $\hat{\sigma}^{2}$ in Equation (9) for comparison. Then correspondingly, we compute the relative MSEs for $\hat{\sigma}^{2}, \hat{\sigma}_{\text {box }}^{2}(d, C)$, and $\hat{\sigma}_{\mathrm{cv}}^{2}$, respectively.

\subsection{Simulation results}

Tables 1-6 list the relative MSEs for the mean functions with jump points, respectively, under the equidistant design. In general, we observe that $\operatorname{MSE}\left(\hat{\sigma}_{\mathrm{CV}}^{2}\right) \bumpeq \operatorname{MSE}\left(\hat{\sigma}_{\text {box }}^{2}\right)<\operatorname{MSE}\left(\hat{\sigma}_{\mathrm{MS}}^{2}\right)<$ $\operatorname{MSE}\left(\hat{\sigma}_{\mathrm{TW}}^{2}\right)$ for small and moderate $\sigma$ values, and $\operatorname{MSE}\left(\hat{\sigma}_{\mathrm{CV}}^{2}\right) \bumpeq \operatorname{MSE}\left(\hat{\sigma}_{\mathrm{box}}^{2}\right) \bumpeq \operatorname{MSE}\left(\hat{\sigma}_{\mathrm{TW}}^{2}\right)<$ $\operatorname{MSE}\left(\hat{\sigma}_{\mathrm{MS}}^{2}\right)$ for large $\sigma$ values. These results show that the proposed adaptive estimators outperform the existing estimators in the presence of jump discontinuities. We also observe that the comparative performance of $\hat{\sigma}_{\text {box }}^{2}\left(d_{t}, 2\right), \hat{\sigma}_{\text {box }}^{2}\left(d_{t}, 3\right), \hat{\sigma}_{\text {box }}^{2}\left(d_{s}, 2\right)$, and $\hat{\sigma}_{\text {box }}^{2}\left(d_{s}, 3\right)$ depends on the smoothness and continuity of the mean function, the sample size, and the signal-to-noise ratio. As reported in Tong and Wang [24], $\hat{\sigma}_{\text {box }}^{2}\left(d_{s}, \cdot\right)$ may not perform well when the sample size is small. As a compromise, $\hat{\sigma}_{\mathrm{CV}}^{2}$ performs well in most settings.

In contrast, we list in Tables 7-9 the relative MSEs for the continuous mean functions $f_{7}(x)$ through $f_{9}(x)$, under the equidistant design. We observe that $\hat{\sigma}_{\text {box }}^{2}, \hat{\sigma}_{\mathrm{CV}}^{2}$, and $\hat{\sigma}_{\mathrm{TW}}^{2}$ perform very similar under various settings. More specifically, we observe that for a continuous mean function, very few $z_{i j}$ values were detected from simulations as outliers. As a consequence, both $\hat{\sigma}_{\text {box }}^{2}\left(d_{t}, 2\right)$ and $\hat{\sigma}_{\text {box }}^{2}\left(d_{t}, 3\right)$ perform essentially the same as $\hat{\sigma}_{\mathrm{TW}}^{2}\left(m_{t}\right)$, and both $\hat{\sigma}_{\text {box }}^{2}\left(d_{s}, 2\right)$ and $\hat{\sigma}_{\text {box }}^{2}\left(d_{s}, 3\right)$ perform essentially the same as $\hat{\sigma}_{\mathrm{TW}}^{2}\left(m_{s}\right)$. Apart from them, $\hat{\sigma}_{\mathrm{MS}}^{2}$ does not provide a comparable performance. This coincides the observation in Tong et al. [23] that $\hat{\sigma}_{\mathrm{MS}}^{2}$ is worse than $\hat{\sigma}_{\mathrm{TW}}^{2}$ when the mean function is continuous.

Finally, we list in Tables 10-12 the relative MSEs for the settings with non-equidistant designs. Similarly as above, we observe that $\hat{\sigma}_{\text {box }}^{2}$ perform better than $\hat{\sigma}^{2}$ in the presence of jump discontinuities, and their performance are similar when the mean function is continuous. Meanwhile, $\hat{\sigma}_{\mathrm{CV}}^{2}$ performs very well in most settings, especially when the sample size is small.

Table 1. Relative MSEs of various estimators for the mean function $f_{1}(x)=g_{1}(x)+h_{1}(x)$, under equidistant design.

\begin{tabular}{|c|c|c|c|c|c|c|c|c|c|c|}
\hline \multirow[b]{2}{*}{$n$} & \multirow[b]{2}{*}{$\sigma$} & \multicolumn{2}{|c|}{$\hat{\sigma}_{\mathrm{MS}}^{2}$} & \multicolumn{2}{|c|}{$\hat{\sigma}_{\mathrm{TW}}^{2}$} & \multicolumn{4}{|c|}{$\hat{\sigma}_{\text {box }}^{2}$} & \multirow[b]{2}{*}{$\hat{\sigma}_{\mathrm{CV}}^{2}$} \\
\hline & & $L_{t}$ & $L_{S}$ & $m_{t}$ & $m_{s}$ & $\left(d_{t}, 2\right)$ & $\left(d_{t}, 3\right)$ & $\left(d_{s}, 2\right)$ & $\left(d_{s}, 3\right)$ & \\
\hline \multirow[t]{5}{*}{30} & 0.2 & 65.5 & 34.6 & 513 & 1368 & 1.64 & 1.60 & 1.67 & 1.79 & 1.62 \\
\hline & 0.5 & 20.8 & 9.58 & 16.8 & 39.0 & 2.41 & 7.78 & 6.12 & 24.4 & 2.82 \\
\hline & 1 & 13.2 & 4.94 & 2.87 & 4.15 & 2.48 & 2.83 & 3.40 & 4.13 & 2.87 \\
\hline & 2 & 11.0 & 3.61 & 1.66 & 1.54 & 1.63 & 1.66 & 1.49 & 1.53 & 1.47 \\
\hline & 5 & 10.3 & 3.23 & 1.49 & 1.26 & 1.51 & 1.50 & 1.25 & 1.26 & 1.30 \\
\hline \multirow[t]{5}{*}{100} & 0.2 & 14.3 & 12.0 & 308 & 939 & 1.37 & 1.38 & 1.22 & 1.21 & 1.25 \\
\hline & 0.5 & 5.38 & 3.76 & 9.75 & 26.0 & 1.41 & 2.80 & 1.39 & 5.22 & 1.33 \\
\hline & 1 & 4.05 & 2.52 & 2.02 & 2.92 & 1.71 & 1.98 & 1.99 & 2.82 & 1.59 \\
\hline & 2 & 3.73 & 2.19 & 1.43 & 1.34 & 1.37 & 1.44 & 1.28 & 1.34 & 1.26 \\
\hline & 5 & 3.64 & 2.10 & 1.36 & 1.20 & 1.35 & 1.36 & 1.18 & 1.20 & 1.19 \\
\hline \multirow{5}{*}{500} & 0.2 & 3.85 & 3.92 & 130 & 777 & 1.24 & 1.22 & 1.11 & 1.11 & 1.17 \\
\hline & 0.5 & 2.36 & 1.79 & 4.46 & 20.8 & 1.25 & 1.43 & 1.15 & 1.92 & 1.20 \\
\hline & 1 & 2.15 & 1.47 & 1.41 & 2.29 & 1.25 & 1.38 & 1.35 & 2.13 & 1.32 \\
\hline & 2 & 2.10 & 1.38 & 1.23 & 1.17 & 1.22 & 1.23 & 1.11 & 1.17 & 1.13 \\
\hline & 5 & 2.08 & 1.36 & 1.22 & 1.10 & 1.24 & 1.22 & 1.13 & 1.11 & 1.16 \\
\hline
\end{tabular}


Table 2. Relative MSEs of various estimators for the mean function $f_{2}(x)=g_{2}(x)+h_{1}(x)$, under equidistant design.

\begin{tabular}{|c|c|c|c|c|c|c|c|c|c|c|}
\hline \multirow[b]{2}{*}{$n$} & \multirow[b]{2}{*}{$\sigma$} & \multicolumn{2}{|c|}{$\hat{\sigma}_{\mathrm{MS}}^{2}$} & \multicolumn{2}{|c|}{$\hat{\sigma}_{\mathrm{TW}}^{2}$} & \multicolumn{4}{|c|}{$\hat{\sigma}_{\text {box }}^{2}$} & \multirow[b]{2}{*}{$\hat{\sigma}_{\mathrm{CV}}^{2}$} \\
\hline & & $L_{t}$ & $L_{S}$ & $m_{t}$ & $m_{s}$ & $\left(d_{t}, 2\right)$ & $\left(d_{t}, 3\right)$ & $\left(d_{s}, 2\right)$ & $\left(d_{s}, 3\right)$ & \\
\hline \multirow[t]{5}{*}{30} & 0.2 & 61.1 & 23.2 & 526 & 1509 & 1.78 & 1.75 & 3.49 & 15.2 & 2.71 \\
\hline & 0.5 & 20.8 & 9.40 & 17.1 & 42.6 & 1.90 & 5.64 & 4.50 & 26.7 & 2.66 \\
\hline & 1 & 13.2 & 5.05 & 2.89 & 4.38 & 2.35 & 2.80 & 3.28 & 4.28 & 3.04 \\
\hline & 2 & 11.0 & 3.65 & 1.66 & 1.55 & 1.64 & 1.66 & 1.49 & 1.55 & 1.53 \\
\hline & 5 & 10.3 & 3.24 & 1.49 & 1.26 & 1.51 & 1.50 & 1.26 & 1.26 & 1.27 \\
\hline \multirow[t]{5}{*}{100} & 0.2 & 14.2 & 12.3 & 309 & 961 & 1.39 & 1.40 & 1.28 & 1.27 & 1.37 \\
\hline & 0.5 & 5.36 & 3.81 & 9.81 & 26.5 & 1.39 & 2.29 & 1.25 & 3.73 & 1.28 \\
\hline & 1 & 4.04 & 2.52 & 2.02 & 2.95 & 1.67 & 1.95 & 1.86 & 2.79 & 1.58 \\
\hline & 2 & 6.72 & 2.20 & 1.43 & 1.34 & 1.40 & 1.44 & 1.28 & 1.34 & 1.28 \\
\hline & 5 & 3.64 & 2.10 & 1.36 & 1.20 & 1.36 & 1.37 & 1.18 & 1.20 & 1.20 \\
\hline \multirow[t]{5}{*}{500} & 0.2 & 3.84 & 3.94 & 130 & 780 & 1.24 & 1.22 & 1.12 & 1.13 & 1.18 \\
\hline & 0.5 & 2.36 & 1.79 & 4.47 & 20.9 & 1.24 & 1.39 & 1.14 & 1.62 & 1.22 \\
\hline & 1 & 2.15 & 1.47 & 1.41 & 2.30 & 1.25 & 1.38 & 1.32 & 2.10 & 1.32 \\
\hline & 2 & 2.10 & 1.38 & 1.23 & 1.17 & 1.22 & 1.23 & 1.11 & 1.17 & 1.13 \\
\hline & 5 & 2.08 & 1.36 & 1.22 & 1.11 & 1.24 & 1.22 & 1.13 & 1.11 & 1.16 \\
\hline
\end{tabular}

Table 3. Relative MSEs of various estimators for the mean function $f_{3}(x)=g_{3}(x)+h_{1}(x)$, under equidistant design.

\begin{tabular}{|c|c|c|c|c|c|c|c|c|c|c|}
\hline \multirow[b]{2}{*}{$n$} & \multirow[b]{2}{*}{$\sigma$} & \multicolumn{2}{|c|}{$\hat{\sigma}_{\mathrm{MS}}^{2}$} & \multicolumn{2}{|c|}{$\hat{\sigma}_{\mathrm{TW}}^{2}$} & \multicolumn{4}{|c|}{$\hat{\sigma}_{\text {box }}^{2}$} & \multirow[b]{2}{*}{$\hat{\sigma}_{\mathrm{CV}}^{2}$} \\
\hline & & $L_{t}$ & $L_{S}$ & $m_{t}$ & $m_{s}$ & $\left(d_{t}, 2\right)$ & $\left(d_{t}, 3\right)$ & $\left(d_{s}, 2\right)$ & $\left(d_{s}, 3\right)$ & \\
\hline \multirow[t]{5}{*}{30} & 0.2 & 61.4 & 22.7 & 506 & 1297 & 1.70 & 1.95 & 76.1 & 1427 & 1.69 \\
\hline & 0.5 & 20.6 & 9.06 & 16.6 & 37.2 & 3.91 & 11.7 & 24.7 & 37.0 & 4.51 \\
\hline & 1 & 13.2 & 4.90 & 2.86 & 4.05 & 2.60 & 2.83 & 3.81 & 4.05 & 2.71 \\
\hline & 2 & 11.0 & 3.61 & 1.66 & 1.53 & 1.64 & 1.66 & 1.50 & 1.53 & 1.50 \\
\hline & 5 & 10.3 & 3.23 & 1.49 & 1.26 & 1.51 & 1.50 & 1.26 & 1.26 & 1.30 \\
\hline \multirow[t]{5}{*}{100} & 0.2 & 14.3 & 12.0 & 306 & 927 & 1.39 & 1.38 & 1.28 & 1.27 & 1.35 \\
\hline & 0.5 & 5.39 & 3.78 & 9.72 & 25.7 & 1.45 & 3.34 & 1.81 & 9.95 & 1.41 \\
\hline & 1 & 4.05 & 2.52 & 2.01 & 2.90 & 1.72 & 1.98 & 2.15 & 2.86 & 1.60 \\
\hline & 2 & 3.73 & 2.20 & 1.43 & 1.34 & 1.40 & 1.44 & 1.29 & 1.34 & 1.26 \\
\hline & 5 & 3.64 & 2.10 & 1.36 & 1.20 & 1.35 & 1.36 & 1.18 & 1.20 & 1.19 \\
\hline \multirow{5}{*}{500} & 0.2 & 3.85 & 3.93 & 130 & 775 & 1.23 & 1.22 & 1.11 & 1.12 & 1.17 \\
\hline & 0.5 & 2.36 & 1.79 & 4.46 & 20.7 & 1.25 & 1.44 & 1.16 & 2.33 & 1.23 \\
\hline & 1 & 2.15 & 1.47 & 1.41 & 2.29 & 1.25 & 1.38 & 1.38 & 2.15 & 1.33 \\
\hline & 2 & 2.10 & 1.38 & 1.23 & 1.17 & 1.22 & 1.23 & 1.11 & 1.17 & 1.13 \\
\hline & 5 & 2.08 & 1.36 & 1.22 & 1.11 & 1.24 & 1.22 & 1.13 & 1.11 & 1.16 \\
\hline
\end{tabular}

\section{Case study}

For illustration, we apply the proposed methods to a real data example. The data were reported in Cobb [2] on the annual volume of discharge in the Nile River from 1895 to 1934. In Figure 3, we find several observations with large variation and we suspect that the mean function might contain jump discontinuities. For this data with $n=40$ observations, we choose $L_{t}=m_{t}=\left\lfloor n^{1 / 3}\right\rfloor=3$ and $L_{s}=m_{s}=\left\lfloor n^{1 / 2}\right\rfloor=6$ for $\hat{\sigma}_{\mathrm{MS}}^{2}$ and $\hat{\sigma}_{\mathrm{TW}}^{2}$, respectively. Here, $\lfloor a\rfloor$ denotes the largest integer smaller than or equal to $a$. For the proposed methods, correspondingly we choose $d_{t}=$ $\left(m_{t} / n\right)^{2}=0.075^{2}$ and $d_{s}=\left(m_{s} / n\right)^{2}=0.15^{2}$. The estimated residual variances are as follows: $\hat{\sigma}_{\mathrm{MS}}^{2}\left(L_{t}\right)=126.9, \hat{\sigma}_{\mathrm{MS}}^{2}\left(L_{s}\right)=47.7 ; \hat{\sigma}_{\mathrm{TW}}^{2}\left(m_{t}\right)=119.9$ and $\hat{\sigma}_{\mathrm{TW}}^{2}\left(m_{s}\right)=144.8 ; \hat{\sigma}_{\text {box }}^{2}\left(d_{t}, 2\right)=126.1$, 
Table 4. Relative MSEs of various estimators for the mean function $f_{4}(x)=g_{1}(x)+h_{2}(x)$, under equidistant design.

\begin{tabular}{|c|c|c|c|c|c|c|c|c|c|c|}
\hline \multirow[b]{2}{*}{$n$} & \multirow[b]{2}{*}{$\sigma$} & \multicolumn{2}{|c|}{$\hat{\sigma}_{\mathrm{MS}}^{2}$} & \multicolumn{2}{|c|}{$\hat{\sigma}_{\mathrm{TW}}^{2}$} & \multicolumn{4}{|c|}{$\hat{\sigma}_{\text {box }}^{2}$} & \multirow[b]{2}{*}{$\hat{\sigma}_{\mathrm{CV}}^{2}$} \\
\hline & & $L_{t}$ & $L_{s}$ & $m_{t}$ & $m_{s}$ & $\left(d_{t}, 2\right)$ & $\left(d_{t}, 3\right)$ & $\left(d_{s}, 2\right)$ & $\left(d_{s}, 3\right)$ & \\
\hline \multirow[t]{5}{*}{30} & 0.2 & 88.2 & 47.3 & 1217 & 3177 & 1.69 & 5.45 & 3177 & 3177 & 1.69 \\
\hline & 0.5 & 24.6 & 12.3 & 36.1 & 86.7 & 9.65 & 24.2 & 86.7 & 86.7 & 18.3 \\
\hline & 1 & 14.3 & 5.80 & 4.39 & 7.48 & 3.88 & 4.30 & 7.41 & 7.48 & 4.52 \\
\hline & 2 & 11.3 & 3.85 & 1.84 & 1.84 & 1.79 & 1.84 & 1.81 & 1.84 & 1.71 \\
\hline & 5 & 10.3 & 3.28 & 1.52 & 1.28 & 1.53 & 1.52 & 1.28 & 1.29 & 1.30 \\
\hline \multirow[t]{5}{*}{100} & 0.2 & 20.3 & 16.0 & 309 & 2274 & 1.35 & 1.15 & 1.23 & 1.28 & 1.29 \\
\hline & 0.5 & 6.51 & 4.62 & 9.75 & 60.8 & 1.37 & 1.18 & 4.37 & 29.0 & 2.09 \\
\hline & 1 & 1.33 & 2.76 & 1.94 & 5.21 & 1.34 & 1.33 & 4.00 & 5.17 & 2.24 \\
\hline & 2 & 3.80 & 2.27 & 1.34 & 1.51 & 1.36 & 1.17 & 1.44 & 1.51 & 1.34 \\
\hline & 5 & 3.65 & 2.12 & 1.32 & 1.20 & 1.38 & 1.22 & 1.19 & 1.20 & 1.19 \\
\hline \multirow[t]{5}{*}{500} & 0.2 & 4.82 & 5.52 & 318 & 1902 & 1.24 & 1.22 & 1.13 & 1.13 & 1.17 \\
\hline & 0.5 & 2.51 & 2.08 & 9.37 & 49.8 & 1.28 & 2.10 & 1.39 & 7.98 & 1.37 \\
\hline & 1 & 2.18 & 1.56 & 1.74 & 4.13 & 1.34 & 1.68 & 2.23 & 3.92 & 1.56 \\
\hline & 2 & 2.10 & 1.41 & 1.26 & 1.29 & 1.23 & 1.26 & 1.18 & 1.29 & 1.18 \\
\hline & 5 & 2.08 & 1.36 & 1.22 & 1.11 & 1.24 & 1.22 & 1.12 & 1.11 & 1.16 \\
\hline
\end{tabular}

Table 5. Relative MSEs of various estimators for the mean function $f_{5}(x)=g_{2}(x)+h_{2}(x)$, under equidistant design.

\begin{tabular}{|c|c|c|c|c|c|c|c|c|c|c|}
\hline \multirow[b]{2}{*}{$n$} & \multirow[b]{2}{*}{$\sigma$} & \multicolumn{2}{|c|}{$\hat{\sigma}_{\mathrm{MS}}^{2}$} & \multicolumn{2}{|c|}{$\hat{\sigma}_{\mathrm{TW}}^{2}$} & \multicolumn{4}{|c|}{$\hat{\sigma}_{\text {box }}^{2}$} & \multirow[b]{2}{*}{$\hat{\sigma}_{\mathrm{CV}}^{2}$} \\
\hline & & $L_{t}$ & $L_{S}$ & $m_{t}$ & $m_{s}$ & $\left(d_{t}, 2\right)$ & $\left(d_{t}, 3\right)$ & $\left(d_{s}, 2\right)$ & $\left(d_{s}, 3\right)$ & \\
\hline \multirow[t]{5}{*}{30} & 0.2 & 82.8 & 30.0 & 1270 & 3770 & 1.94 & 4.73 & 3739 & 3770 & 1.93 \\
\hline & 0.5 & 24.5 & 11.5 & 37.5 & 102 & 6.38 & 21.7 & 93.4 & & 22.0 \\
\hline & 1 & 14.3 & 5.78 & 4.47 & 8.39 & 3.70 & 4.36 & 7.95 & 8.37 & 5.64 \\
\hline & 2 & 11.3 & 3.85 & 1.84 & 1.89 & 1.80 & 1.84 & 1.82 & 1.89 & 1.72 \\
\hline & 5 & 10.3 & 3.28 & 1.52 & 1.28 & 1.53 & 1.52 & 1.27 & 1.29 & 1.30 \\
\hline \multirow[t]{5}{*}{100} & 0.2 & 20.2 & 16.0 & 751 & 2358 & 1.43 & 1.44 & 1.34 & 1.66 & 1.42 \\
\hline & 0.5 & 6.50 & 4.66 & 21.3 & 63.0 & 1.78 & 5.65 & 3.28 & 25.8 & 1.80 \\
\hline & 1 & 4.33 & 2.76 & 2.80 & 5.35 & 2.11 & 2.72 & 3.70 & 5.26 & 2.23 \\
\hline & 2 & 3.79 & 2.27 & 1.50 & 1.51 & 1.45 & 1.50 & 1.43 & 1.51 & 1.35 \\
\hline & 5 & 3.65 & 2.12 & 1.37 & 1.20 & 1.36 & 1.37 & 1.19 & 1.20 & 1.20 \\
\hline \multirow[t]{5}{*}{500} & 0.2 & 4.82 & 5.55 & 318 & 1913 & 1.24 & 1.23 & 1.14 & 1.15 & 1.20 \\
\hline & 0.5 & 2.51 & 2.09 & 9.38 & 50.2 & 1.28 & 1.98 & 1.30 & 6.16 & 1.34 \\
\hline & 1 & 2.18 & 1.56 & 1.74 & 4.15 & 1.34 & 1.68 & 2.09 & 3.89 & 1.52 \\
\hline & 2 & 2.10 & 1.41 & 1.26 & 1.29 & 1.23 & 1.26 & 1.18 & 1.29 & 1.19 \\
\hline & 5 & 2.08 & 1.36 & 1.22 & 1.11 & 1.24 & 1.22 & 1.12 & 1.11 & 1.17 \\
\hline
\end{tabular}

$\hat{\sigma}_{\text {box }}^{2}\left(d_{t}, 3\right)=119.9, \hat{\sigma}_{\text {box }}^{2}\left(d_{s}, 2\right)=137.2, \hat{\sigma}_{\text {box }}^{2}\left(d_{s}, 3\right)=144.8$, and $\hat{\sigma}_{\text {CV }}^{2}=119.9$. We note that for a standard with $C=3$, no outliers were identified so that $\hat{\sigma}_{\text {box }}^{2}\left(d_{t}, 3\right)=\hat{\sigma}_{\text {TW }}^{2}\left(m_{t}\right)=119.9$ and $\hat{\sigma}_{\text {box }}^{2}\left(d_{s}, 3\right)=\hat{\sigma}_{\mathrm{TW}}^{2}\left(m_{s}\right)=144.8$. In addition, the $\mathrm{CV}$ method suggests to take $C=3$ with a bandwidth at $d_{t}$ and that results in the variance estimate as 119.9. Recall that the suggested value of $\sigma^{2}$ is 125 in Cobb [2]. We conclude that our pairwise regression method performs at least as well as the least-squares estimator $\sigma_{\mathrm{TW}}^{2}$. Nevertheless, the estimator $\sigma_{\mathrm{MS}}^{2}$ is very sensitive to the choice of the bandwidth and so is less reliable. 
Table 6. Relative MSEs of various estimators for the mean function $f_{6}(x)=g_{3}(x)+h_{2}(x)$, under equidistant design.

\begin{tabular}{|c|c|c|c|c|c|c|c|c|c|c|}
\hline \multirow[b]{2}{*}{$n$} & \multirow[b]{2}{*}{$\sigma$} & \multicolumn{2}{|c|}{$\hat{\sigma}_{\mathrm{MS}}^{2}$} & \multicolumn{2}{|c|}{$\hat{\sigma}_{\mathrm{TW}}^{2}$} & \multicolumn{4}{|c|}{$\hat{\sigma}_{\text {box }}^{2}$} & \multirow[b]{2}{*}{$\hat{\sigma}_{\mathrm{CV}}^{2}$} \\
\hline & & $L_{t}$ & $L_{S}$ & $m_{t}$ & $m_{s}$ & $\left(d_{t}, 2\right)$ & $\left(d_{t}, 3\right)$ & $\left(d_{s}, 2\right)$ & $\left(d_{s}, 3\right)$ & \\
\hline \multirow[t]{5}{*}{30} & 0.2 & 82.9 & 30.9 & 1190 & 2902 & 4.47 & 117 & 2902 & 2902 & 12.2 \\
\hline & 0.5 & 24.3 & 11.5 & 35.5 & 79.7 & 15.8 & 30.7 & 79.7 & 79.7 & 23.1 \\
\hline & 1 & 14.3 & 5.78 & 4.35 & 7.07 & 4.11 & 4.32 & 7.06 & 7.07 & 4.21 \\
\hline & 2 & 11.3 & 3.85 & 1.84 & 1.82 & 1.81 & 1.84 & 1.79 & 1.82 & 1.70 \\
\hline & 5 & 10.3 & 3.28 & 1.52 & 1.28 & 1.54 & 1.52 & 1.28 & 1.29 & 1.30 \\
\hline \multirow[t]{5}{*}{100} & 0.2 & 20.3 & 15.9 & 740 & 2229 & 1.41 & 1.40 & 1.30 & 9.69 & 1.39 \\
\hline & 0.5 & 6.52 & 4.64 & 21.1 & 59.7 & 2.41 & 8.89 & 9.79 & 45.3 & 2.47 \\
\hline & 1 & 4.34 & 2.76 & 2.78 & 5.14 & 2.25 & 2.76 & 4.30 & 5.13 & 2.26 \\
\hline & 2 & 3.80 & 2.27 & 1.49 & 1.50 & 1.46 & 1.50 & 1.45 & 1.50 & 1.33 \\
\hline & 5 & 3.66 & 2.12 & 1.37 & 1.20 & 1.35 & 1.37 & 1.18 & 1.20 & 1.19 \\
\hline \multirow[t]{5}{*}{500} & 0.2 & 4.83 & 5.53 & 317 & 1895 & 1.23 & 1.22 & 1.12 & 1.23 & 1.18 \\
\hline & 0.5 & 2.51 & 2.09 & 9.36 & 49.7 & 1.28 & 2.19 & 1.56 & 10.6 & 1.39 \\
\hline & 1 & 2.18 & 1.56 & 1.74 & 4.12 & 1.35 & 1.68 & 2.34 & 3.95 & 1.56 \\
\hline & 2 & 2.10 & 1.41 & 1.26 & 1.29 & 1.23 & 1.26 & 1.18 & 1.29 & 1.18 \\
\hline & 5 & 2.08 & 1.36 & 1.22 & 1.11 & 1.24 & 1.22 & 1.12 & 1.11 & 1.16 \\
\hline
\end{tabular}

Table 7. Relative MSEs of various estimators for the mean function $f_{7}(x)=g_{1}(x)+h_{3}(x)$, under equidistant design.

\begin{tabular}{|c|c|c|c|c|c|c|c|c|c|c|}
\hline \multirow[b]{2}{*}{$n$} & \multirow[b]{2}{*}{$\sigma$} & \multicolumn{2}{|c|}{$\hat{\sigma}_{\mathrm{MS}}^{2}$} & \multicolumn{2}{|c|}{$\hat{\sigma}_{\mathrm{TW}}^{2}$} & \multicolumn{4}{|c|}{$\hat{\sigma}_{\text {box }}^{2}$} & \multirow[b]{2}{*}{$\hat{\sigma}_{\mathrm{CV}}^{2}$} \\
\hline & & $L_{t}$ & $L_{S}$ & $m_{t}$ & $m_{s}$ & $\left(d_{t}, 2\right)$ & $\left(d_{t}, 3\right)$ & $\left(d_{s}, 2\right)$ & $\left(d_{s}, 3\right)$ & \\
\hline \multirow[t]{5}{*}{30} & 0.2 & 10.2 & 3.54 & 1.53 & 1.64 & 1.56 & 1.53 & 1.72 & 1.64 & 1.58 \\
\hline & 0.5 & 10.1 & 3.21 & 1.49 & 1.27 & 1.52 & 1.49 & 1.26 & 1.27 & 1.39 \\
\hline & 1 & 10.1 & 3.17 & 1.48 & 1.25 & 1.51 & 1.49 & 1.24 & 1.25 & 1.40 \\
\hline & 2 & 10.1 & 3.16 & 1.48 & 1.24 & 1.51 & 1.49 & 1.25 & 1.25 & 1.24 \\
\hline & 5 & 10.1 & 3.16 & 1.48 & 1.24 & 1.51 & 1.49 & 1.25 & 1.25 & 1.29 \\
\hline \multirow[t]{5}{*}{100} & 0.2 & 3.65 & 2.10 & 1.35 & 1.21 & 1.34 & 1.36 & 1.21 & 1.21 & 1.25 \\
\hline & 0.5 & 3.64 & 2.08 & 1.35 & 1.19 & 1.35 & 1.36 & 1.18 & 1.19 & 1.18 \\
\hline & 1 & 3.63 & 2.08 & 1.35 & 1.19 & 1.35 & 1.36 & 1.18 & 1.19 & 1.17 \\
\hline & 2 & 3.63 & 2.08 & 1.35 & 1.19 & 1.35 & 1.36 & 1.18 & 1.19 & 1.19 \\
\hline & 5 & 3.63 & 2.08 & 1.35 & 1.19 & 1.35 & 1.36 & 1.18 & 1.19 & 1.18 \\
\hline \multirow[t]{5}{*}{500} & 0.2 & 2.08 & 1.35 & 1.22 & 1.11 & 1.24 & 1.22 & 1.12 & 1.11 & 1.17 \\
\hline & 0.5 & 2.08 & 1.35 & 1.22 & 1.11 & 1.25 & 1.22 & 1.14 & 1.11 & 1.16 \\
\hline & 1 & 2.08 & 1.35 & 1.22 & 1.12 & 1.25 & 1.22 & 1.14 & 1.12 & 1.16 \\
\hline & 2 & 2.08 & 1.35 & 1.22 & 1.12 & 1.25 & 1.22 & 1.15 & 1.12 & 1.10 \\
\hline & 5 & 2.08 & 1.35 & 1.22 & 1.12 & 1.25 & 1.22 & 1.15 & 1.12 & 1.15 \\
\hline
\end{tabular}

\section{Discussion}

In this paper, we first introduced a pairwise regression method for estimating $\sigma^{2}$ in nonparametric regression models with continuous mean function. We further extended the pairwise regression method to model (1) with jump discontinuities via adaptation. As already mentioned in Section 1, the proposed adaptive method generalizes the existing methods from different points of view and has several important merits. In particular, our adaptive method turns out to be superior for its flexibility in eliminating the effect of potential jumps in the mean function and for its applicability in both equally and unequally design settings. In addition, compared with the residual-based 
Table 8. Relative MSEs of various estimators for the mean function $f_{8}(x)=g_{2}(x)+h_{3}(x)$, under equidistant design.

\begin{tabular}{|c|c|c|c|c|c|c|c|c|c|c|}
\hline \multirow[b]{2}{*}{$n$} & \multirow[b]{2}{*}{$\sigma$} & \multicolumn{2}{|c|}{$\hat{\sigma}_{\mathrm{MS}}^{2}$} & \multicolumn{2}{|c|}{$\hat{\sigma}_{\mathrm{TW}}^{2}$} & \multicolumn{4}{|c|}{$\hat{\sigma}_{\mathrm{box}}^{2}$} & \multirow[b]{2}{*}{$\hat{\sigma}_{\mathrm{CV}}^{2}$} \\
\hline & & $L_{t}$ & $L_{s}$ & $m_{t}$ & $m_{s}$ & $\overline{\left(d_{t}, 2\right)}$ & $\left(d_{t}, 3\right)$ & $\left(d_{s}, 2\right)$ & $\left(d_{s}, 3\right)$ & \\
\hline \multirow[t]{5}{*}{30} & 0.2 & 11.1 & 9.56 & 1.62 & 2.23 & 1.74 & 1.64 & 2.43 & 2.23 & 1.68 \\
\hline & 0.5 & 10.3 & 3.75 & 1.50 & 1.34 & 1.49 & 1.50 & 1.36 & 1.34 & 1.46 \\
\hline & 1 & 10.2 & 3.27 & 1.48 & 1.26 & 1.49 & 1.48 & 1.27 & 1.26 & 1.37 \\
\hline & 2 & 10.1 & 3.19 & 1.48 & 1.25 & 1.51 & 1.48 & 1.26 & 1.25 & 1.23 \\
\hline & 5 & 10.1 & 3.17 & 1.48 & 1.24 & 1.51 & 1.48 & 1.25 & 1.25 & 1.29 \\
\hline \multirow[t]{5}{*}{100} & 0.2 & 3.68 & 2.56 & 1.37 & 1.25 & 1.35 & 1.38 & 1.33 & 1.25 & 1.35 \\
\hline & 0.5 & 3.63 & 2.11 & 1.36 & 1.19 & 1.34 & 1.36 & 1.17 & 1.19 & 1.18 \\
\hline & 1 & 3.63 & 2.08 & 1.36 & 1.19 & 1.34 & 1.36 & 1.18 & 1.19 & 1.17 \\
\hline & 2 & 3.63 & 2.08 & 1.35 & 1.19 & 1.35 & 1.36 & 1.18 & 1.19 & 1.20 \\
\hline & 5 & 3.63 & 2.08 & 1.35 & 1.19 & 1.35 & 1.36 & 1.18 & 1.19 & 1.17 \\
\hline \multirow[t]{5}{*}{500} & 0.2 & 2.08 & 1.36 & 1.22 & 1.12 & 1.24 & 1.22 & 1.11 & 1.12 & 1. \\
\hline & 0.5 & 2.08 & 1.35 & 1.22 & 1.12 & 1.24 & 1.22 & 1.14 & 1.12 & 1.16 \\
\hline & 1 & 2.08 & 1.35 & 1.22 & 1.12 & 1.24 & 1.22 & 1.15 & 1.12 & 1.16 \\
\hline & 2 & 2.08 & 1.35 & 1.22 & 1.12 & 1.24 & 1.22 & 1.15 & 1.12 & 1.09 \\
\hline & 5 & 2.08 & 1.35 & 1.22 & 1.12 & 1.25 & 1.22 & 1.15 & 1.12 & 1.16 \\
\hline
\end{tabular}

Table 9. Relative MSEs of various estimators for the mean function $f_{9}(x)=g_{3}(x)+h_{3}(x)$, under equidistant design.

\begin{tabular}{|c|c|c|c|c|c|c|c|c|c|c|}
\hline \multirow[b]{2}{*}{$n$} & \multirow[b]{2}{*}{$\sigma$} & \multicolumn{2}{|c|}{$\hat{\sigma}_{\mathrm{MS}}^{2}$} & \multicolumn{2}{|c|}{$\hat{\sigma}_{\mathrm{TW}}^{2}$} & \multicolumn{4}{|c|}{$\hat{\sigma}_{\text {box }}^{2}$} & \multirow[b]{2}{*}{$\hat{\sigma}_{\mathrm{CV}}^{2}$} \\
\hline & & $L_{t}$ & $L_{S}$ & $m_{t}$ & $m_{s}$ & $\left(d_{t}, 2\right)$ & $\left(d_{t}, 3\right)$ & $\left(d_{s}, 2\right)$ & $\left(d_{s}, 3\right)$ & \\
\hline \multirow[t]{5}{*}{30} & 0.2 & 10.4 & 8.34 & 1.61 & 2.26 & 1.68 & 1.61 & 2.48 & 2.26 & 1.70 \\
\hline & 0.5 & 10.1 & 3.48 & 1.50 & 1.32 & 1.54 & 1.50 & 1.34 & 1.32 & 1.44 \\
\hline & 1 & 10.1 & 3.21 & 1.49 & 1.26 & 1.51 & 1.50 & 1.24 & 1.26 & 1.42 \\
\hline & 2 & 10.1 & 3.17 & 1.48 & 1.25 & 1.51 & 1.49 & 1.24 & 1.25 & 1.24 \\
\hline & 5 & 10.1 & 3.16 & 1.48 & 1.24 & 1.50 & 1.49 & 1.24 & 1.25 & 1.28 \\
\hline \multirow[t]{5}{*}{100} & 0.2 & 3.70 & 2.30 & 1.35 & 1.24 & 1.36 & 1.36 & 1.29 & 1.24 & 1.34 \\
\hline & 0.5 & 3.65 & 2.09 & 1.35 & 1.20 & 1.34 & 1.35 & 1.19 & 1.20 & 1.19 \\
\hline & 1 & 3.64 & 2.08 & 1.35 & 1.19 & 1.34 & 1.35 & 1.18 & 1.19 & 1.18 \\
\hline & 2 & 3.64 & 2.08 & 1.35 & 1.19 & 1.34 & 1.36 & 1.18 & 1.19 & 1.19 \\
\hline & 5 & 3.63 & 2.08 & 1.35 & 1.19 & 1.35 & 1.36 & 1.18 & 1.19 & 1.18 \\
\hline \multirow[t]{5}{*}{500} & 0.2 & 2.08 & 1.36 & 1.22 & 1.11 & 1.24 & 1.22 & 1.10 & 1.11 & 1.16 \\
\hline & 0.5 & 2.08 & 1.35 & 1.22 & 1.11 & 1.25 & 1.22 & 1.13 & 1.11 & 1.16 \\
\hline & 1 & 2.08 & 1.35 & 1.22 & 1.11 & 1.25 & 1.22 & 1.14 & 1.12 & 1.16 \\
\hline & 2 & 2.08 & 1.35 & 1.22 & 1.12 & 1.25 & 1.22 & 1.14 & 1.12 & 1.09 \\
\hline & 5 & 2.08 & 1.35 & 1.22 & 1.12 & 1.25 & 1.22 & 1.15 & 1.12 & 1.15 \\
\hline
\end{tabular}

estimators, our method provides a direct way to estimate the residual variance without the estimations of mean function and jump points. In conclusion, we recommend to use the estimator $\hat{\sigma}_{\mathrm{CV}}^{2}$ in practice. More work, though, is needed for demonstrating the theoretical results of our proposed estimators.

The proposed method can be readily extended to higher-dimensional regression models. Consider, for instance, the following bivariate nonparametric regression model with jump discontinuities

$$
y_{i}=g^{*}\left(x_{1 i}, x_{2 i}\right)+h^{*}\left(x_{1 i}, x_{2 i}\right)+\varepsilon_{i}, \quad 1 \leq i \leq n .
$$


Table 10. Relative MSEs of various estimators for the mean function $f_{3}(x)=g_{3}(x)+h_{1}(x)$, under non-equidistant design.

\begin{tabular}{|c|c|c|c|c|c|c|c|c|}
\hline \multirow[b]{2}{*}{$n$} & \multirow[b]{2}{*}{$\sigma$} & \multicolumn{2}{|c|}{$\hat{\sigma}^{2}$} & \multicolumn{4}{|c|}{$\hat{\sigma}_{\text {box }}^{2}$} & \multirow[b]{2}{*}{$\hat{\sigma}_{\mathrm{CV}}^{2}$} \\
\hline & & $d_{t}$ & $d_{s}$ & $\left(d_{t}, 2\right)$ & $\left(d_{t}, 3\right)$ & $\left(d_{s}, 2\right)$ & $\left(d_{s}, 3\right)$ & \\
\hline \multirow[t]{5}{*}{30} & 0.2 & 1174 & 2875 & 34.7 & 34.7 & 659 & 1647 & 50.2 \\
\hline & 0.5 & 35.2 & 81.6 & 7.41 & 21.7 & 49.1 & 76.4 & 8.94 \\
\hline & 1 & 4.28 & 7.45 & 3.74 & 4.17 & 6.89 & 7.40 & 4.16 \\
\hline & 2 & 1.85 & 1.92 & 1.81 & 1.85 & 1.88 & 1.92 & 1.84 \\
\hline & 5 & 1.56 & 1.35 & 1.57 & 1.57 & 1.35 & 1.35 & 1.42 \\
\hline \multirow[t]{5}{*}{100} & 0.2 & 654 & 1726 & 1.60 & 1.61 & 1.57 & 1.59 & 1.57 \\
\hline & 0.5 & 18.8 & 46.2 & 1.64 & 4.31 & 2.30 & 16.7 & 1.72 \\
\hline & 1 & 2.63 & 4.23 & 1.99 & 2.54 & 2.97 & 4.12 & 2.00 \\
\hline & 2 & 1.54 & 1.50 & 1.50 & 1.54 & 1.45 & 1.50 & 1.43 \\
\hline & 5 & 1.45 & 1.31 & 1.45 & 1.45 & 1.30 & 1.31 & 1.36 \\
\hline \multirow[t]{5}{*}{500} & 0.2 & 252 & 1233 & 1.43 & 1.44 & 1.33 & 1.33 & 1.40 \\
\hline & 0.5 & 7.90 & 33.1 & 1.43 & 1.80 & 1.36 & 3.48 & 1.41 \\
\hline & 1 & 1.86 & 3.34 & 1.52 & 1.82 & 1.94 & 3.17 & 1.58 \\
\hline & 2 & 1.46 & 1.44 & 1.44 & 1.46 & 1.36 & 1.43 & 1.44 \\
\hline & 5 & 1.43 & 1.30 & 1.43 & 1.43 & 1.30 & 1.30 & 1.38 \\
\hline
\end{tabular}

Table 11. Relative MSEs of various estimators for the mean function $f_{6}(x)=g_{3}(x)+h_{2}(x)$, under non-equidistant design.

\begin{tabular}{|c|c|c|c|c|c|c|c|c|}
\hline \multirow[b]{2}{*}{$n$} & \multirow[b]{2}{*}{$\sigma$} & \multicolumn{2}{|c|}{$\hat{\sigma}^{2}$} & \multicolumn{4}{|c|}{$\hat{\sigma}_{\text {box }}^{2}$} & \multirow[b]{2}{*}{$\hat{\sigma}_{\mathrm{CV}}^{2}$} \\
\hline & & $d_{t}$ & $d_{s}$ & $\left(d_{t}, 2\right)$ & $\left(d_{t}, 3\right)$ & $\left(d_{s}, 2\right)$ & $\left(d_{s}, 3\right)$ & \\
\hline \multirow{5}{*}{30} & 0.2 & 2435 & 6727 & 624 & 827 & 6411 & 6701 & 853 \\
\hline & 0.5 & 70.0 & 182 & 43.1 & 60.7 & 181 & 182 & 62.4 \\
\hline & 1 & 6.99 & 14.4 & 6.38 & 6.94 & 14.4 & 14.4 & 7.06 \\
\hline & 2 & 2.17 & 2.59 & 2.14 & 2.17 & 2.55 & 2.59 & 2.47 \\
\hline & 5 & 1.61 & 1.43 & 1.62 & 1.61 & 1.42 & 1.43 & 1.51 \\
\hline \multirow[t]{5}{*}{100} & 0.2 & 1477 & 4583 & 1.60 & 1.62 & 121 & 296 & 1.59 \\
\hline & 0.5 & 41.0 & 121 & 4.98 & 20.9 & 47.4 & 103 & 5.82 \\
\hline & 1 & 4.28 & 9.15 & 3.41 & 4.19 & 8.12 & 9.13 & 3.71 \\
\hline & 2 & 1.72 & 1.87 & 1.66 & 1.72 & 1.79 & 1.87 & 1.67 \\
\hline & 5 & 1.48 & 1.34 & 1.46 & 1.48 & 1.32 & 1.34 & 1.37 \\
\hline \multirow[t]{5}{*}{500} & 0.2 & 567 & 3458 & 1.44 & 1.45 & 1.32 & 1.33 & 1.40 \\
\hline & 0.5 & 16.1 & 90.2 & 1.56 & 3.93 & 3.01 & 27.5 & 1.66 \\
\hline & 1 & 2.41 & 6.97 & 1.75 & 2.35 & 4.20 & 6.77 & 1.89 \\
\hline & 2 & 1.51 & 1.68 & 1.46 & 1.51 & 1.52 & 1.68 & 1.53 \\
\hline & 5 & 1.44 & 1.31 & 1.43 & 1.44 & 1.29 & 1.31 & 1.38 \\
\hline
\end{tabular}

We can define $d_{i j}$ by $d_{i j}=\sqrt{\left(x_{1 i}-x_{1 j}\right)^{2}+\left(x_{2 i}-x_{2 j}\right)^{2}}$ or by $d_{i j}=\left|x_{1 i}-x_{1 j}\right|+\left|x_{1 i}-x_{1 j}\right|$, and then proceed the estimation similarly as in Sections 2.2 and 2.3. Further research is necessary to investigate the practical rules for the corresponding adaptive method as well as to evaluate its finite-sample performance. Furthermore, recall that the proposed method in this paper is restricted to a constant residual variance assumption. As this may not be realistic in applications, it should be of interest to propose new pairwise regression methods for estimating the variance function in regression models with jump discontinuities. 
Table 12. Relative MSEs of various estimators for the mean function $f_{9}(x)=g_{3}(x)+h_{3}(x)$, under non-equidistant design.

\begin{tabular}{|c|c|c|c|c|c|c|c|c|}
\hline \multirow[b]{2}{*}{$n$} & \multirow[b]{2}{*}{$\sigma$} & \multicolumn{2}{|c|}{$\hat{\sigma}^{2}$} & \multicolumn{4}{|c|}{$\hat{\sigma}_{\text {box }}^{2}$} & \multirow[b]{2}{*}{$\hat{\sigma}_{\mathrm{CV}}^{2}$} \\
\hline & & $d_{t}$ & $d_{s}$ & $\overline{\left(d_{t}, 2\right)}$ & $\left(d_{t}, 3\right)$ & $\left(d_{s}, 2\right)$ & $\overline{\left(d_{s}, 3\right)}$ & \\
\hline \multirow[t]{5}{*}{30} & 0.2 & 2.29 & 3.55 & 2.21 & 2.28 & 3.39 & 3.54 & 2.22 \\
\hline & 0.5 & 1.63 & 1.51 & 1.62 & 1.63 & 1.49 & 1.51 & 1.52 \\
\hline & 1 & 1.56 & 1.35 & 1.57 & 1.56 & 1.34 & 1.35 & 1.40 \\
\hline & 2 & 1.54 & 1.31 & 1.55 & 1.54 & 1.30 & 1.31 & 1.38 \\
\hline & 5 & 1.53 & 1.30 & 1.55 & 1.54 & 1.30 & 1.30 & 1.29 \\
\hline \multirow[t]{5}{*}{100} & 0.2 & 1.58 & 1.50 & 1.56 & 1.58 & 1.47 & 1.50 & 1.53 \\
\hline & 0.5 & 1.48 & 1.33 & 1.47 & 1.48 & 1.33 & 1.33 & 1.38 \\
\hline & 1 & 1.46 & 1.32 & 1.45 & 1.46 & 1.32 & 1.32 & 1.38 \\
\hline & 2 & 1.46 & 1.32 & 1.44 & 1.46 & 1.32 & 1.32 & 1.38 \\
\hline & 5 & 1.45 & 1.32 & 1.44 & 1.45 & 1.33 & 1.32 & 1.38 \\
\hline \multirow[t]{5}{*}{500} & 0.2 & 1.43 & 1.32 & 1.43 & 1.43 & 1.30 & 1.32 & 1.37 \\
\hline & 0.5 & 1.43 & 1.30 & 1.43 & 1.43 & 1.30 & 1.30 & 1.38 \\
\hline & 1 & 1.43 & 1.30 & 1.43 & 1.43 & 1.30 & 1.30 & 1.38 \\
\hline & 2 & 1.43 & 1.30 & 1.43 & 1.43 & 1.31 & 1.30 & 1.38 \\
\hline & 5 & 1.43 & 1.29 & 1.43 & 1.43 & 1.31 & 1.29 & 1.38 \\
\hline
\end{tabular}

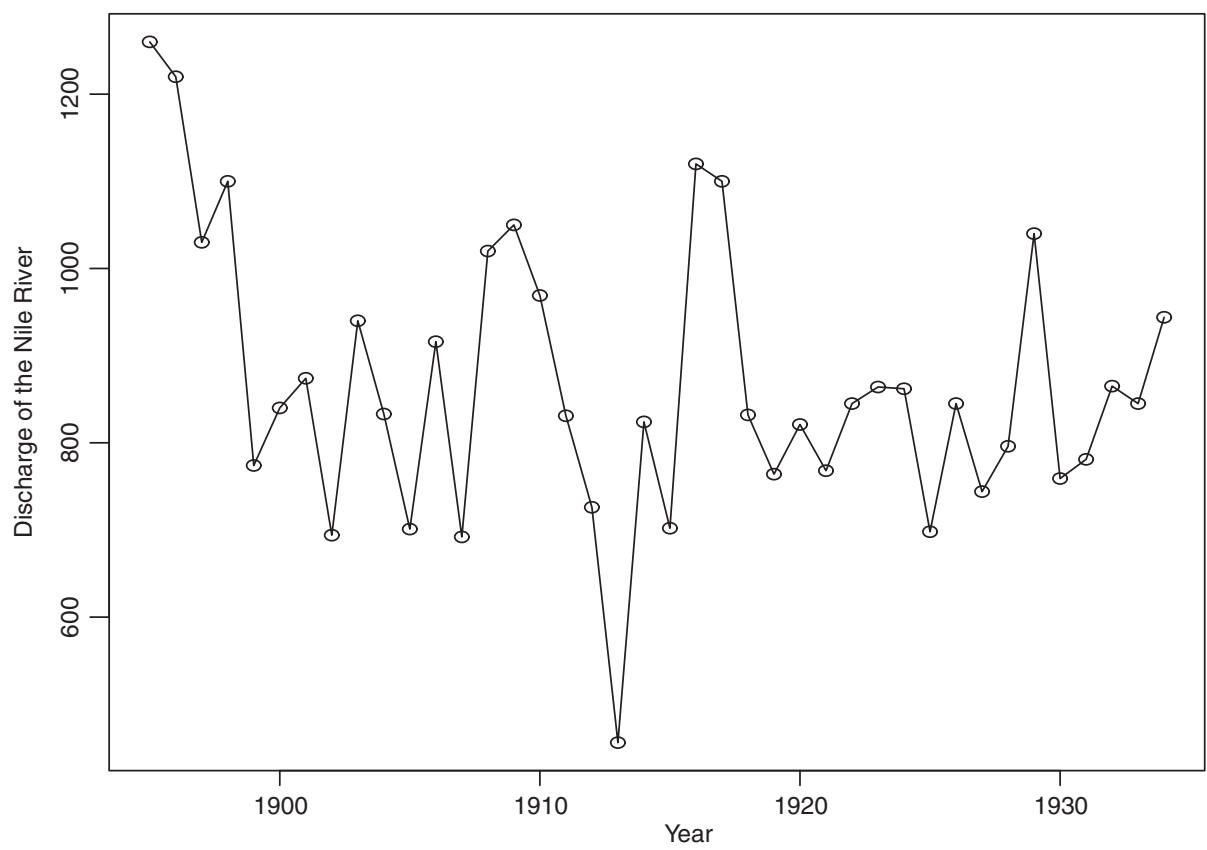

Figure 3. The Nile discharge data from 1895 to 1934.

\section{Acknowledgements}

This research was supported by Hong Kong RGC grant HKBU202711, and Hong Kong Baptist University grants FRG2/10$11 / 020$ and FRG2/11-12/110. The authors thank the editor, the associate editor, and two referees for their helpful comments and suggestions that have substantially improved the paper. 


\section{References}

[1] R.L. Brown, J. Durbin, and J.M. Evans, Techniques for testing the constancy of regression relationships over time, J. R. Stat. Soc. Ser. B 37 (1975), pp. 149-192.

[2] G.W. Cobb, The problem of the Nile: Conditional solution to a changepoint problem, Biometrika 65 (1978), pp. 243251.

[3] J. Du and A. Schick, A covariate-matched estimator of the error variance in nonparametric regression, J. Nonparametric Stat. 21 (2009), pp. 263-285.

[4] R.L. Eubank and P.L. Speckman, Nonparametric estimation of functions with jump discontinuities, IMS Lecture Notes: Change-Point Probl. 23 (1994), pp. 130-144.

[5] T. Gasser, L. Sroka, and C. Jennen-Steinmetz, Residual variance and residual pattern in nonlinear regression, Biometrika 73 (1986), pp. 625-633.

[6] P. Hall, J.W. Kay, and D.M. Titterington, Asymptotically optimal difference-based estimation of variance in nonparametric regression, Biometrika 77 (1990), pp. 521-528.

[7] P. Hall and J.S. Marron, On variance estimation in nonparametric regression, Biometrika 77 (1990), pp. $415-419$.

[8] P. Hall and D.M. Titterington, Edge-preserving and peak-preserving smoothing, Technometrics 34 (1992), pp. 429440.

[9] D.V. Hinkley, Inference about the intersection in two-phase regression, Biometrika 56 (1969), pp. $495-504$.

[10] H.J. Kim and D. Siegmund, The likelihood ratio test for a change-point in simple linear regression, Biometrika 76 (1989), pp. 409-423.

[11] C.R. Loader, Change point estimation using nonparametric regression, Ann. Stat. 24 (1996), pp. 1667-1678.

[12] J. McDonald and A. Owen, Smoothing with split linear fits, Technometrics 28 (1986), pp. 195-208.

[13] H. Müller, Change-points in nonparametric regression analysis, Ann. Stat. 20 (1992), pp. 737-761.

[14] H. Müller and U. Stadtmüller, Discontinuous versus smooth regression, Ann. Stat. 27 (1999), pp. $299-337$.

[15] U. Müller, A. Schick, and W. Wefelmeyer, Estimating the error variance in nonparametric regression by a covariatematched U-statistic, Statistics 37 (2003), pp. 179-188.

[16] J. von Neumann, Distribution of the ratio of the mean squared successive difference to the variance, Ann. Math. Stat. 12 (1941), pp. 367-395.

[17] P. Qiu, Image Processing and Jump Regression Analysis, John Wiley \& Sons, New York, 2005.

[18] P. Qiu and D. Hawkins, A rank-based multivariate cusum procedure, Technometrics 43 (2001), pp. 120-132.

[19] P. Qiu and B. Yandell, A local polynomial jump detection algorithm in nonparametric regression, Technometrics 40 (1998), pp. 141-152.

[20] J.A. Rice, Bandwidth choice for nonparametric regression, Ann. Stat. 12 (1984), pp. 1215-1230.

[21] C.H. Sim, F.F. Gan, and T.C. Chang, Outlier labeling with boxplot procedures, J. Am. Stat. Assoc. 100 (1994), pp. 642-652.

[22] T. Tong, A. Liu, and Y. Wang, Relative errors of difference-based variance estimators in nonparametric regression, Commun. Stat. Theory Methods 37 (2008), pp. 2890-2902.

[23] T. Tong, Y. Ma, and Y. Wang, Optimal variance estimation without estimating the mean function, Bernoulli (in press).

[24] T. Tong and Y. Wang, Estimating residual variance in nonparametric regression using least squares, Biometrika 92 (2005), pp. 821-830.

[25] Y. Wang, Jump and sharp cusp detection by wavelets, Biometrika 82 (1995), pp. 385-397.

[26] J.S. Wu and C.K. Chu, Kernel type estimators of jump points and values of a regression function, Ann. Stat. 21 (1993), pp. $1545-1566$.

[27] J.S. Wu and C.K. Chu, Nonparametric function estimation and bandwidth selection for discontinuous regression functions, Stat. Sin. 3 (1993), pp. 557-576. 